\title{
Descrição de uma nova espécie de Chlorotabanus (Insecta, Diptera, Tabanidae) da Região Neotropical
}

\author{
Tiago K. Krolow \& Augusto L. Henriques
}

Coordenação de Pesquisas em Entomologia, Instituto Nacional de Pesquisas da Amazônia, Caixa Postal 478, 69011-970 Manaus, AM, Brasil. (tkkrolow@gmail.com; loureiro@inpa.gov.br)

\begin{abstract}
Description of a new species of Chlorotabanus (Insecta, Diptera, Tabanidae) from Neotropical Region. We described a new species of Tabanidae (Diptera), Chlorotabanus flagellatus sp. nov., distributed in the Amazon region, in the States of Amazonas and Pará. Diagnosis, discussion and illustrations of external and internal characters are provided.
\end{abstract}

KEYWORDS. Amazon region, Chlorotabanus, new species, Tabanidae, taxonomy.

RESUMO. É descrita uma nova espécie de Tabanidae (Diptera), Chlorotabanus flagellatus sp. nov., distribuída na região amazônica, Estados do Amazonas e Pará. Diagnose, discussão e ilustrações dos caracteres externos e internos são fornecidas.

PALAVRAS-CHAVE. Amazônia, Chlorotabanus, espécie nova, Tabanidae, taxonomia.

O gênero Chlorotabanus foi proposto por LuTz (1913), para Tabanus mexicanus Linnaeus, 1758. Na última revisão do gênero, PHILIP \& FAIRCHILD (1956) propuseram Cryptotylus Lutz como subgênero de Chlorotabanus, com cinco espécies para o subgênero nominal e outras cinco para o subgênero Cryptotylus. FAIRCHILD (1961) descreve Chlorotabanus (Chlorotabanus) leucochlorus. Em seguida Cryptotylus é novamente elevado ao status de gênero (FAIRCHILD, 1969). Posteriormente, WILKERSON (1979) descreve a última espécie do gênero. Atualmente, há sete espécies de Chlorotabanus descritas para as Américas, sendo encontradas do sul dos Estados Unidos à Argentina (FAIRCHILD \& BURGER, 1994; BURGER, 1995).

As espécies do gênero são facilmente distinguíveis de outros gêneros de Tabanidae, devido à coloração esverdeada característica (quando em vida), ausência do calo frontal, flagelo antenal com no máximo um ângulo obtuso no dorso da placa basal, labela esclerotinizada e asa hialina ou com pequenas manchas escuras nos intercruzamentos das veias. Além disso, seu hábito é comumente associado à atividade noturna ou crepuscular, já que raramente são observados durante o dia (RAFAel \& ChARlwood, 1981).

O estudo de material das coleções amazônicas permitiu a determinação de uma nova espécie. Dessa forma, o trabalho tem como objetivo descrevê-la, apresentando os principais caracteres externos e internos, de forma detalhada possibilitando a diferenciação das demais espécies.

\section{MATERIAL E MÉTODOS}

Os espécimes foram obtidos através do empréstimo de material das seguintes instituições: Instituto Nacional de Pesquisas da Amazônia (INPA, Manaus), Coleção Zoológica Paulo Bührnheim (CZPB, Manaus) e Museu Paraense Emílio Goeldi (MPEG, Belém). Parátipos estão depositados nas instituições de empréstimo e nas seguintes coleções: California Academy of Sciences (CAS, San Francisco), Florida State Collection of
Arthropods (FSCA, Gainesville) e Museu de Zoologia da Universidade de São Paulo (MZSP, São Paulo).

A terminologia morfológica externa segue McAlpine (1981) e FAIRChiLd (1986), e para a terminália Sinclair et al. (1993). Para a dissecação das terminálias foi utilizado o protocolo de Cumming (1992). Foram feitas medições de fronte e antena para a utilização de índices. Para fronte, foram utilizados os índices frontal (I.F.) e de divergência (I.D.) conforme FAIRCHILD (1985), enquanto para antenas, foram estimados outros dois índices, o índice de placa basal (I.P.B.), que consiste da maior altura da placa basal dividida pelo comprimento da mesma, e o índice do flagelo (I.Flg.), que é o comprimento da placa basal dividido pelo comprimento do estilo. As variações de medidas e índices são apresentadas do valor mínimo para o máximo, onde $\mathrm{X}$ e $\mathrm{N}$ significam respectivamente média e número de indivíduos observados. Para a melhor visualização do tegumento torácico, é utilizada a deposição de uma pequena gota de álcool.

Informações adicionais entre colchetes foram inseridas para completar os dados das etiquetas do material examinado.

\section{Chlorotabanus flagellatus sp. nov. (Figs. 1-14)}

Chlorotabanus inanis; Henriques \& Gorayeb, 1993:9 (part., non Chlorotabanus inanis (Fabricius, 1787)); Henriques, 1995:69 (part., non C. inanis (Fabricius, 1787))

Diagnose (fêmea). Comprimento total entre 13,5-17 $\mathrm{mm}$, corpo de amarelo-escuro a esverdeado, com pilosidade dourado-alaranjada. Olhos vermelho-escuros, com fronte estreita e alongada (I.F. > 8.0). Antena com placa basal alongada, esta, cerca de duas vezes e meia o comprimento do estilo.

Holótipo fêmea (Fig. 1). Comprimento do corpo sem antenas $15,8 \mathrm{~mm}$; comprimento da asa $13,4 \mathrm{~mm}$; largura da asa $3,9 \mathrm{~mm}$.

Cabeça. Olhos nus, vermelho-escuros. Tubérculo ocelar ausente, em seu lugar, um pequeno triângulo de 
pêlos dourados. Fronte (Fig. 2) estreita e convergente na base; índice frontal 9.0; índice de divergência 1.6; pruinosidade castanho-amarelada e pilosidade dourada, com pêlos cobrindo toda a fronte exceto na região mediana inferior. Subcalo, gena, parafaciália e face com pruinosidade idêntica a da fronte. Subcalo com grande área nua, exceto nas proximidades da parafaciália com pêlos amarelos e esparsos. Gena, parafaciália e face com pilosidade amarela. Palpo amarelo com pilosidade dourada. Teca da probóscide esclerotinizada, amarelada e com tamanho subigual ao comprimento do palpo. Labela esclerotinizada, com coloração verde-escuro. Antena (Fig.

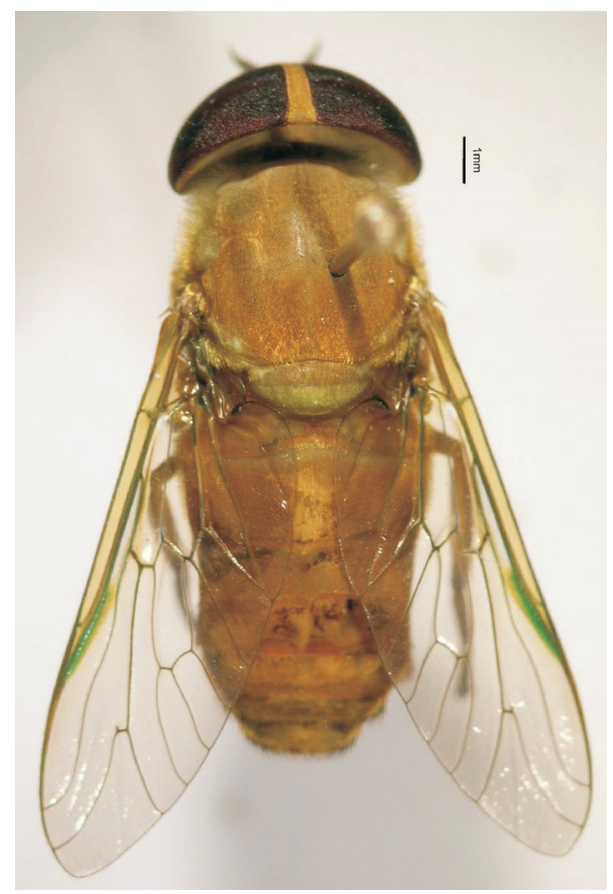

Figura 1. Chlorotabanus flagellatus sp. nov., holótipo fêmea, vista dorsal: habitus.

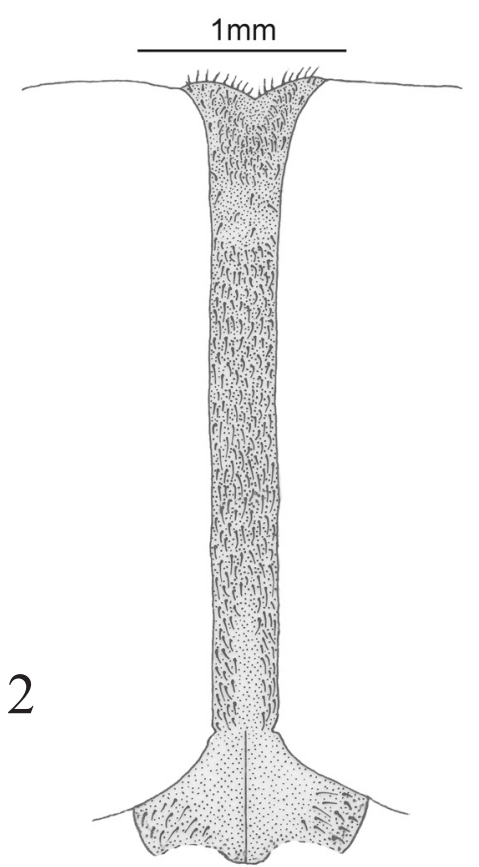

3). Escapo e pedicelo amarelo-esverdeados com pêlos dourados, flagelo verde escuro, exceto pelo último anulus amarelado. Flagelo com placa basal alongada, cerca de duas vezes mais comprida que alta (I.P.B. = 1.9). Estilo aproximadamente duas vezes e meia menor que o comprimento da placa basal (I.Flg. $=2.7)$.

Tórax. Escuto com tegumento alaranjado, coberto por uma camada de pruína branca (vistas dorsal e pósterodorsal) ou alaranjada (vistas lateral e ântero-dorsal), e uma camada pilosa dourado-alaranjada. Escutelo com pruinosidade branca (vista ântero-dorsal) ou ausente (vistas póstero-dorsal e dorsal), camada pilosa concolor ao escuto. Lóbo pós-pronotal, notopleura e calo pósalar com pruinosidade branca em vista lateral e dorsal. Pleura com pruinosidade branca e pilosidade dourada, com maior densidade de pêlos nas proximidades da inserção alar. Pernas delgadas, coxas com pruinosidade branca e pilosidade amarela; trocânteres e fêmures castanho-amarelados com pilosidade dourada, tíbias anterior e média, esverdeadas com pilosidade dourada, tíbia posterior castanho-amarelada, coberta de pêlos negros na face posterior nos $2 / 3$ apicais, face anterior com pêlos dourados. Asa (Fig. 4) hialina, exceto pelo escurecimento das células basal costal, costal, subcostal (área central com mancha hialina) e basal radial (nas proximidades da veia radial), e pelo pterostigma verdeescuro. Incisão alular com mancha escura. Veia costa cerdosa alaranjada, com algumas cerdas escuras esparsas. Veia $\mathrm{R}_{2+3}$ com sinuosidade proeminente, encontrando a veia costa distante da veia $R_{1}$ e próxima da veia $R_{4}$. Pterostigma verde-escuro. Veias transversais mais escurecidas que as longitudinais, amareladas.

Abdômen. Amarelo-escuro, coberto por uma pilosidade dourada, exceto a partir do quinto tergito, onde aparecem faixas distais transversais de pêlos negros. Esternitos amarelo-escuro cobertos por pêlos dourados.

Terminália. Esternito oito e gonapófises (Fig. 5) vista ventral; tergitos nove e dez, cercos e hipoprocto (Fig. 6) vista dorsal; forquilha genital (Fig. 7) e espermateca (Fig. 8) vista dorsal.

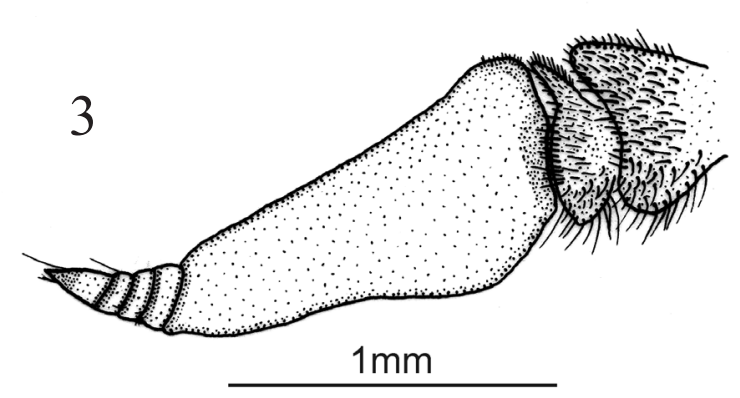

Figuras 2, 3. Chlorotabanus flagellatus sp. nov., holótipo fêmea: 2, fronte, vista frontal; 3, antena esquerda, vista lateral. 
Esternito oito coberto de pêlos, com uma forte reentrância na margem anterior; margens laterais arredondadas encontrando a gonapófises. Gonapófises coberta de pêlos, com uma leve reentrância na margem posterior; presença de uma projeção triangular na região mediana anterior, que se estende à porção posterior do esternito oito (vista dorsal). Tergitos nove, dez e cercos, cobertos de pêlos. Tergito nove com tamanho reduzido em relação ao décimo tergito; afilado nas margens externa anterior e interna posterior. Tergito dez com formato subretangular; margem externa arredondada na base, com região posterior levemente acuminada. Cercos com margens internas e externas arredondadas, ambas convergindo em direção ao ápice posterior, proporcionando um formato levemente acuminado. Hipoprocto quase totalmente encoberto pelos cercos em vista dorsal, apenas com sua região mediana visível; ápice e base arredondados. Forquilha genital com margem

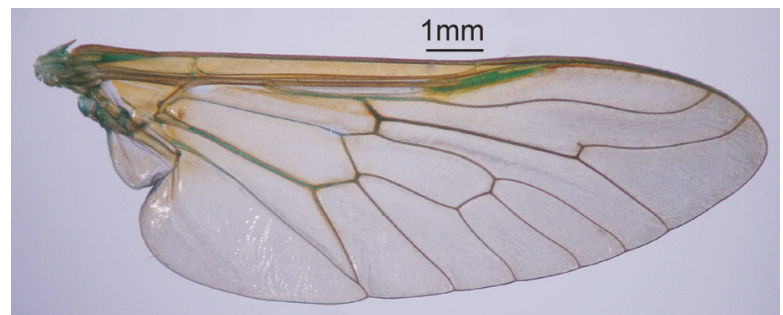

Figura 4. Chlorotabanus flagellatus sp. nov., parátipo fêmea: asa direita, vista dorsal.
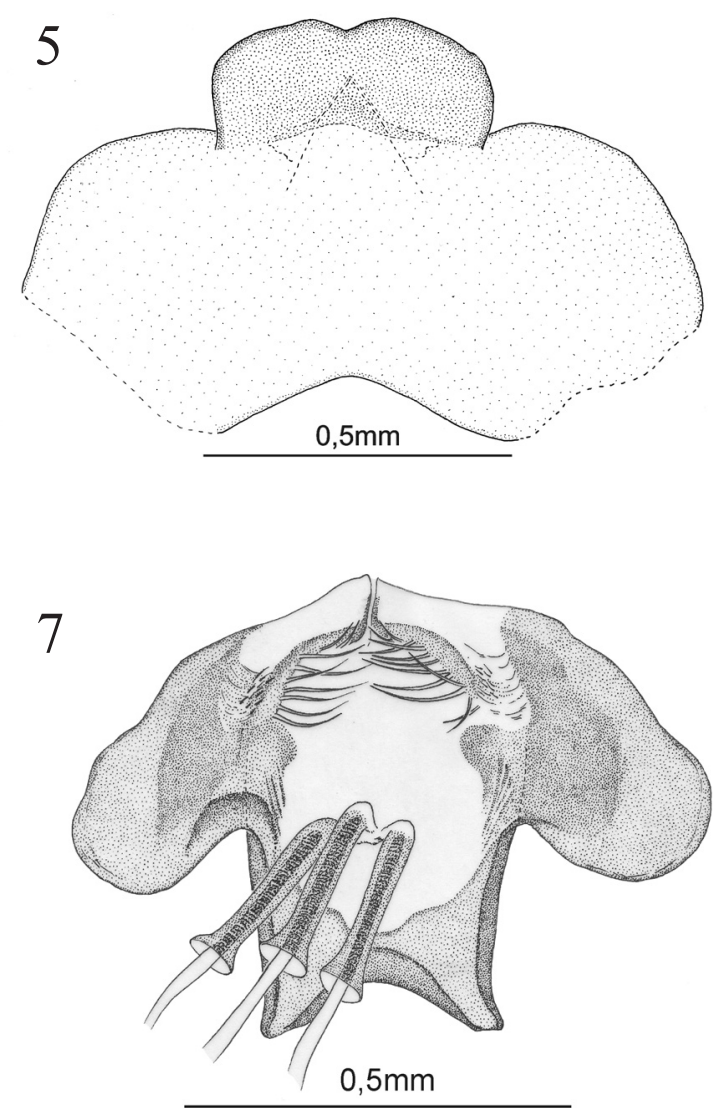

anterior escavada na região central; dois lobos posteriores laterais, paralelos e bem desenvolvidos; região apical com dois tufos fortes de cerdas paralelas, convergindo no ápice posterior. Espermateca tubular e translúcida, exceto pela cápsula espermática ovóide e escura.

Variações: os parátipos variam no tamanho do corpo sem antenas de 13,5-17,0 $\mathrm{mm}(X=15,1 \mathrm{~mm}, \mathrm{~N}=30)$; comprimento da asa $11,2-13,4 \mathrm{~mm}(X=12,4 \mathrm{~mm}, \mathrm{~N}=30)$; largura da asa $3,4-4,3 \mathrm{~mm}(\mathrm{X}=4,0 \mathrm{~mm}, \mathrm{~N}=30)$; I.F. 8.0$10.4(\mathrm{X}=9.2, \mathrm{~N}=30) ;$ I.D. $1.3-2.0(\mathrm{X}=1.6, \mathrm{~N}=30) ;$ I.P.B. $1.7-2.2(\mathrm{X}=2.0, \mathrm{~N}=30)$; I.Flg. $2 \cdot 0-3 \cdot 4(\mathrm{X}=2.5, \mathrm{~N}=30)$.

Além do corpo, variando de amarelo-escuro a esverdeado, diversas estruturas também apresentam variação de cor entre os espécimes observados: olhos variando de tons acinzentados até vermelho-escuro; labela de castanho-escuro, verde-escuro a preto; antena de amarelo a verde-escuro, podendo variar nos três segmentos e entre os artículos; pterostigma de castanhoamarelado a verde;

Uma possível explicação para tantas variações é que, as espécies de Chlorotabanus possuem hemolinfa verde, e como a variação de cor muitas vezes está relacionada à distribuição interna dos pigmentos, estes podem ou não colorir partes como antenas, pernas, tórax e abdômen (WilKerson, 1979), levando a inúmeras combinações de coloração ao longo do corpo. Outra variação comumente observada é quanto ao escurecimento dos últimos segmentos abdominais, que,
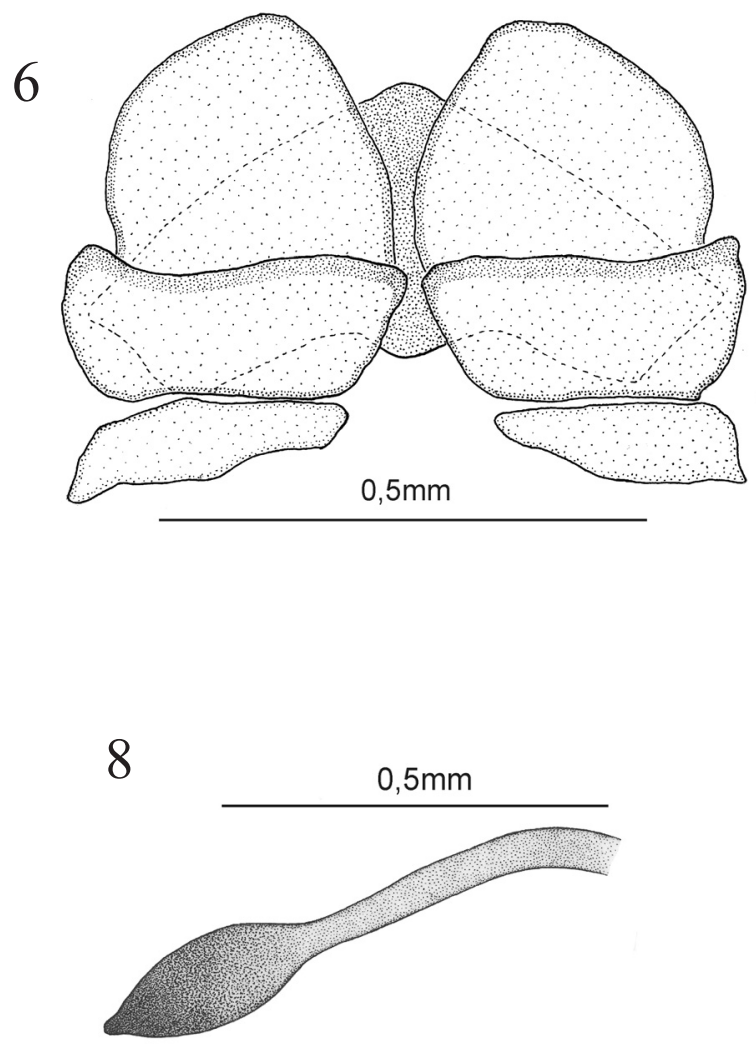

Figuras 5-8. Chlorotabanus flagellatus sp. nov., parátipo fêmea: 5, esternito oito e gonapófises, vista ventral (cerdas não representadas); 6 , tergitos nove e dez, cercos e hipoprocto, vista dorsal (cerdas não representadas); 7, forquilha genital, vista dorsal; 8, espermateca, vista dorsal. 
conforme WILKERSON (1979) pode ser causada por pequenas diferenças de pigmentação externa ou pela presença de um repasto sanguíneo não digerido.

Macho. Comprimento do corpo sem antenas $15,0 \mathrm{~mm}$, variando de $13,6-15,8 \mathrm{~mm}(\mathrm{X}=14,9 \mathrm{~mm}, \mathrm{~N}=13)$; comprimento da asa $11,7 \mathrm{~mm}, 10,6-12,5 \mathrm{~mm}(\mathrm{X}=11,5 \mathrm{~mm}, \mathrm{~N}=13)$; largura da asa 3,8 mm, 3,5-3,9 $\mathrm{mm}(\mathrm{X}=3,6 \mathrm{~mm}, \mathrm{~N}=13)$.

Similar à fêmea, exceto por alguns caracteres como, olho holóptico (Fig. 9), com as facetas dorsais mais desenvolvidas, ocupando aproximadamente $2 / 3$ da região superior do olho e distintamente delimitadas das facetas inferiores e marginais, ambas com tamanho reduzido; tubérculo ocelar ausente, com uma fenda; palpo porrecto, amarelo com pêlos dourados. Antena (Fig. 10) com estilo alongado em relação ao da fêmea, este, quase do tamanho da placa basal, I.Flg. 1.3, variando de 1.0-1.5 (X=1.2, $\mathrm{N}=$ 11). I.P.B. $1.8,1.5-2.0(\mathrm{X}=1.7, \mathrm{~N}=11)$.

Terminália. Epândrio, cercos e hipoprocto (Fig. 11), vista dorsal. Hipândrio + gonocoxitos, gonóstilo e edeago (Fig. 12), vista ventral.

Epândrio, com região posterior dilatada e recoberta por pêlos, sofrendo um estreitamento e a perda da pilosidade a partir do primeiro terço até a extremidade proximal, exceto por dois ou três pêlos esparsos na região proximal. Cercos cobertos de pêlos; região anterior achatada, com um arredondamento das margens laterais internas e um leve afilamento na extremidade posterior, este ultrapassando a margem distal do hipoprocto. Hipoprocto encoberto pelos cercos, com apenas a extremidade médio-apical aparente, esta nua e com um pequeno sulco mediano dando um aspecto de fenda; pêlos visíveis apenas em vista ventral.

Hipândrio + gonocoxitos robusto, mais longo que largo; margem anterior truncada com leve arredondamento nas bases laterais. Hipândrio com a margem posterior fortemente escavada anteriormente; região escavada possui uma fileira de pêlos diminutos. Gonocoxitos tubulares, com tufo de pêlos fortes na margem interna, nas proximidades dos gonóstilos. Apódemas gonocoxais bem desenvolvidos, estreitos e alongados, sofrendo uma torção na região mediana, paralelos, ultrapassando a margem anterior do hipândrio

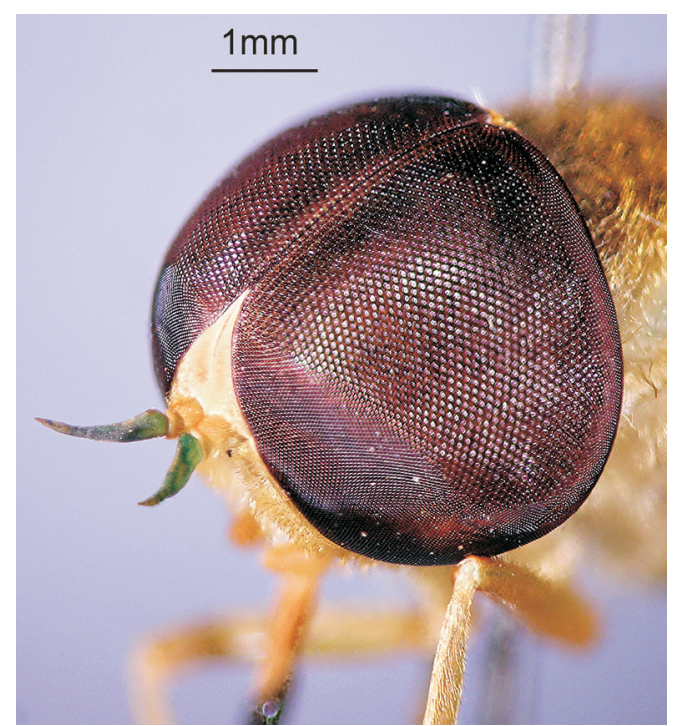

Figura 9. Chlorotabanus flagellatus sp. nov., parátipo macho: cabeça, vista latero-frontal.
+ gonocoxito. Gonóstilos convergentes; tubulares; margem apical média deprimida projetando levemente as margens laterais. Bainha parameral alongada com formato triangular; margem anterior ligada lateralmente aos gonocoxitos; margem posterior afilada alcançando a porção distal dos gonóstilos. Edeago longo em forma de "lança", pouco maior que os apódemas gonocoxais.

Variações. Nove terminálias de macho foram dissecadas apresentando alta similaridade, entretanto, dois espécimes externamente idênticos aos demais, apresentaram variações na forma do hipândrio + gonocoxitos e gonóstilo (Figs. 13, 14, desenho esquemático), vista ventral. A peça, hipândrio + gonocoxitos e gonóstilo (Fig. 13) possui comprimento e largura semelhantes, dando um aspecto quadrado. Abertura do hipândrio + gonocoxitos (vista dorsal) tem formato retangular. Os gonóstilos são largos e truncados. Em outro espécime, hipândrio + gonocoxitos e gonóstilo (Fig. 14) se assemelham muito ao descrito na figura 12, exceto pelo leve estreitamento da base do hipândrio + gonocoxitos, dando um aspecto menos truncado, ou seja, com a base mais arredondada.

Material-tipo. Holótipo $\mathrm{Q}$ : BRASIL. Amazonas: Manaus (AM $010 \mathrm{~km} 54$ BI-2, 02॰45'33''S; 59 51'03'”W, Tapir/anca, Clareira, 5:30-6:00h), 23.IX.1997, R.L.M. Ferreira, A.L. Henriques \& J.F. Vidal leg. Chlorotabanus inanis (sem determinador). Parátipos: idem (Tapir/flanco, Clareira, 18:00-19:00h), ㅇ, 22.VII.1997 (FSCA); idem, (Tapir/ventre, Clareira, 5:30-6:00h), +, 23.IX.1997 (MZSP); idem, (Tapir/flanco, Clareira, 5:30-6:00h), +, (MPEG); idem, (Tapir/anca, Clareira, 17:00-18:00h), \&, 24.IX.1997 (MZSP); idem, (Tapir/dorso, Clareira, 5:30-6:00h), , 11.XI.1997 (MPEG); idem, (Tapir/ventre, Clareira, 17:0018:00h), ㅇ, 12.XI.1997 (INPA); idem, (Tapir/anca, Clareira, 17:0018:00h), +, 12.XI.1997 (INPA); idem, (Tapir/dorso, Clareira, 5:30 6:00h), q, 02.XII.1997 (FSCA); idem, (Tapir/coxa, Clareira, 18:0018:30h), 3ㅇ, 02.XII.1997 (INPA); idem, (Tapir/anca, Clareira, 5:30-6:00h), ㅇ, 03.XII.1997 (MZSP); idem, (Tapir/anca, Clareira, 17:00-18:00h), +, 03.XII.1997 (CZPB); idem, (Tapir/coxa, Clareira, 6:00-7:00h), ㅇ, 21.I.1998 (CZPB); idem, (Tapir/flanco, Clareira, 5:30-6:00h), ㅇ, 03.II.1998 (CAS); idem, (Tapir/anca, Clareira, 6:00-7:00h), 3o, 03.II.1998 (INPA); idem, (Tapir/perna anterior, Clareira, 6:00-7:00h), ㅇ, 03.II.1998 (CAS); idem, ㅇ, 26.XI.1981, J.A. Rafael leg. (CAS); idem, (Bacia do Ig.[arapé] Tinga, isca humana,), , IX.2001, F.C. Costa leg. (INPA); idem, (Igarapé Barro Branco, arm. Malaise 04), 29, 09-12.II.2004, A. Henriques et al. leg. (INPA); idem, (Campus Universitário), 2 , 14.XI.1978, J.A. Rafael leg. (INPA; FSCA); idem, (ZF-2 KM-14, Torre, 023521S-600655W, luz mista/ BL,BLB, lençol, $40 \mathrm{mt}$ altura), +, 18-21.II.2004, J.A. Rafael, C.S. Motta, F.F. Xavier Fo, A.Silva $\mathrm{F}^{\mathrm{o}}$ \& S. Trovisco leg. (INPA); idem, (Armadilha Malaise), o, 16-19.VII.2004, J.A. Rafael, F.F. Xavier $\mathrm{F}^{\circ}$ \& J.M. Ribeiro leg. (MPEG); idem, (Lençol: luz mista e BLB, $40 \mathrm{mts}$ altura), ơ, 1619.VII.2004, J.A. Rafael, C.S. Motta, F.F. Xavier Fo, J.M.F. Ribeiro \& S. Trovisco leg. (INPA); idem, (luz mista/BLB, lençol, $40 \mathrm{mts}$ altura), $q$, 16-19.VII.2004, J.A. Rafael, C.S. Motta, F.F. Xavier Fo, J.M.F. Ribeiro \& S. Trovisco leg. (CAS); idem, (luz mista/BLB, lençol, 36 mts altura), +, 12-15.X.2004, J.A. Rafael, C.S. Motta, F.F. Xavier $\mathrm{F}^{\mathrm{o}}$, A.Silva $\mathrm{F}^{\mathrm{o}}$ \& S. Trovisco leg. (FSCA); idem, (Lençol, luz mista e BLB, 35 mts altura), ơ, 13-16.XI.2004, F.F. Xavier Fo, A.R. Ururahy, F. Godoi \& S. Trovisco leg. (FSCA); idem, (armadilha luz móvel), ㅇ, 01.XI.2005, J.A. Rafael, F.F. Xavier Fo, R. Machado, A.A. Agudelo \& Y.K. Dantas leg. (MZSP); idem, (Estrada ZF-2, armadilha luz móvel), 30', 01.XI.2005, J.A. Rafael, F.F. Xavier Fo, R. Machado, A.A. Agudelo \& Y.K. Dantas leg. (INPA); idem, (BR174, Faz. Porto Alegre, Malaise), +, VII.2002, L. Aquino leg. (FSCA); idem, (F.[azenda] Esteio, R. 1401, km-27 ZF-3, Malaise), +, 05-20.IV.1995, L.E.F.R.Silva leg. (MPEG); AM $240(\mathrm{~km} \mathrm{24,}$ $2^{\circ} 00^{\prime} 55^{\prime} \mathrm{S}$; 59॰49'40'”W, Luz mista/mercúrio), , o', VIII.2005, Xavier-Filho leg. (INPA); Rio Urubu (02 ${ }^{\circ} 10^{\prime} \mathrm{S}$; $\left.59^{\circ} 49^{\prime} \mathrm{W}\right)$, ㅇ, 13 14.III.1983, P. Bührnhein, N. Otaviano \& S. Leite leg. (CZPB); Parque Nacional do Jaú (Rio Carabinani, Igarapé Preguição), , , 2029.VI.1994, L. Aquino leg. (INPA); Itacoatiara (Madeireira Mil, 024510S; 583911W, armadilha luminosa móvel, 05-06hs), +, $2 \sigma^{\prime}$, 29-30.XI.2005, J.A. Rafael, R.J.P. Machado \& A.Silva Fo. leg. 
(INPA); idem, O’, 29-30.XI.2005, J.A. Rafael, R.J.P. Machado \& A.Silva $F^{\circ}$. leg. (MZSP); idem, ơ, 29-30.XI.2005, J.A. Rafael, R.J.P. Machado \& A.Silva F ${ }^{\circ}$. leg. (CAS); idem, O', 29-30.XI.2005, J.A. Rafael, R.J.P. Machado \& A.Silva $\mathrm{F}^{\circ}$. leg. (MPEG); Novo Airão (ramal do Olimpio, 02³9'23"S; 60 55'44"W, luz mista de mercúrio), O', 03-15.VII.1997, P.F. Bührnhein, N.O. Aguiar \& N.F. Fé leg. (CZPB); idem, O', 03-15.VII.1997, P.F. Bührnhein, N.O. Aguiar \& N.F. Fé leg. (INPA); Pará: Benevides (PA-408 km 06), , 21.XI.1980, I.S. Gorayeb \& F.F. Ramos leg. (INPA); idem, 18.III.1981, I.S. Gorayeb leg. (INPA); idem, (cavalo), + 28.VII.1982, R.B. Neto leg. (MPEG).

Material adicional examinado. BRASIL Amazonas: Manaus (AM $010 \mathrm{~km} 54 \mathrm{BI}-2,02^{\circ} 45^{\prime} 33^{\prime \prime} \mathrm{S}$; 59 51'03'W, Centro Instrução do CIGS, em lona azul, 18:08h), , 08.IV.1997, R.L.M. Ferreira, A.L. Henriques \& J.F. Vidal leg. (INPA); idem, (Tapir/ escápula, Clareira, 17:00-18:00h), 29, 12.XI.1997 (INPA); idem, (Tapir/flanco, Clareira, 18:00-18:30h), , 21.I.1998 (INPA); idem, (Reserva Ducke),, , 4.VI.1976, A. B. Anderson leg.. Chlorotabanus sp., det. N.D. Penny (INPA); idem, 29, 26.XI.1981, J.A. Rafael leg. (INPA); idem, (26 km, NE, armadilha suspensa 10 metros), ㅇ,

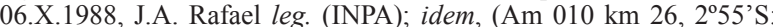
$59^{\circ} 59^{\prime} \mathrm{W}, 06-$ Malaise clareira), , 07. 07..2001, R.L.M. Ferreira et al. leg. Chlorotabanus inanis (sem determinador) (INPA); idem, (Igarapé Acará Branco, coleta manual), 29, 09-13.XI.2007, A. S B. Souza leg. (INPA); idem, (ZF-2, Torre $45 \mathrm{mts}$ altura, luz mista/ mercúrio), 29, 7,8.I.1997, R.L. Ferreira leg. Chlorotabanus inanis, A.L. Henriques, 1997 det. (INPA); Itacoatiara (Madeireira Mil, 024510S-583911W, armadilha luminosa móvel, 19-20hs), $29,29-$ 30.XI.2005, J.A. Rafael, R.J.P. Machado \& A.Silva $F^{\circ}$. leg. (INPA); Novo Airão (Ramal do Olimpio, 02 ${ }^{\circ} 39^{\prime} 23^{\prime \prime} \mathrm{S}$; 6055'44"W, luz mista de mercúrio), ㅇ, 03-15.VII.1997, P.F. Bührnhein, N.O. Aguiar

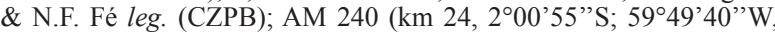
Luz mista/mercúrio), ㅇ, VIII.2005, Xavier-Filho leg. (INPA); Parque Nacional do Jaú (Igarapé Miratuca, 1 ${ }^{\circ} 57$ '08'S; 61 ${ }^{\circ} 49^{\prime} 19^{\prime \prime}$ W, Malaise),, , R. Andreaze, W. Costa \& L. Aquino leg. (INPA); Rio Juruá (Minerauzinho, 033485S; 665915W, luz mista de mercúrio), , 25.I.1996, P.Bührnhein \& N.Otaviano leg. (CZPB); Rio Urubu (02 ${ }^{\circ} 10^{\prime}$ 'S; $\left.59^{\circ} 48^{\prime} \mathrm{W}\right)$, + , 18-19.IX.1982, P. Bührnhein leg. (CZPB).

Etimologia. Do Latim flagellum, relacionado à placa basal alongada do flagelo antenal.

Discussão. O gênero Chlorotabanus é muito bem
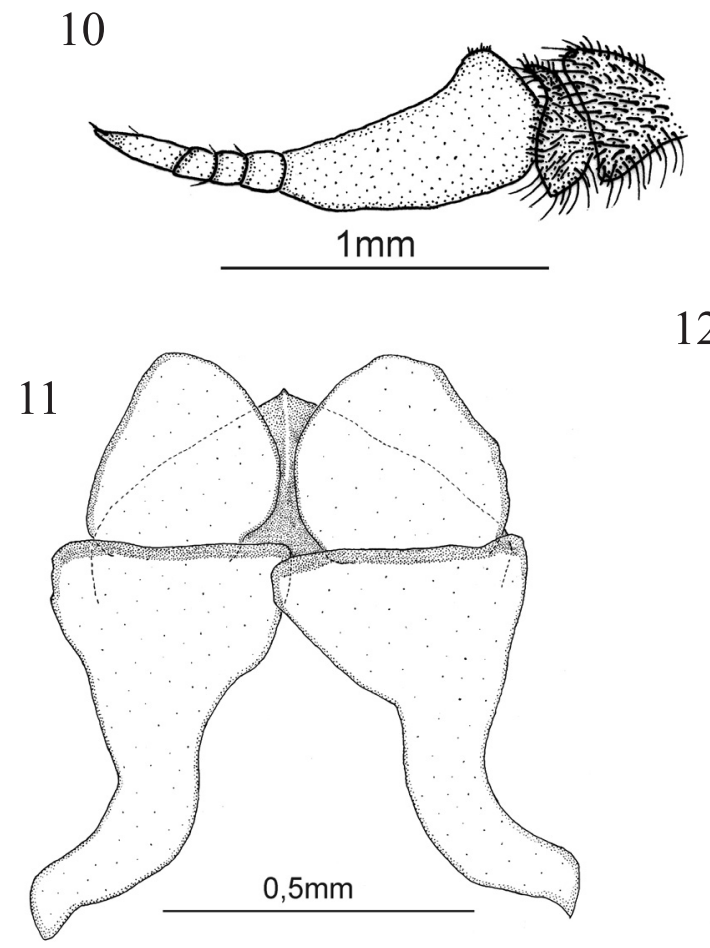

12
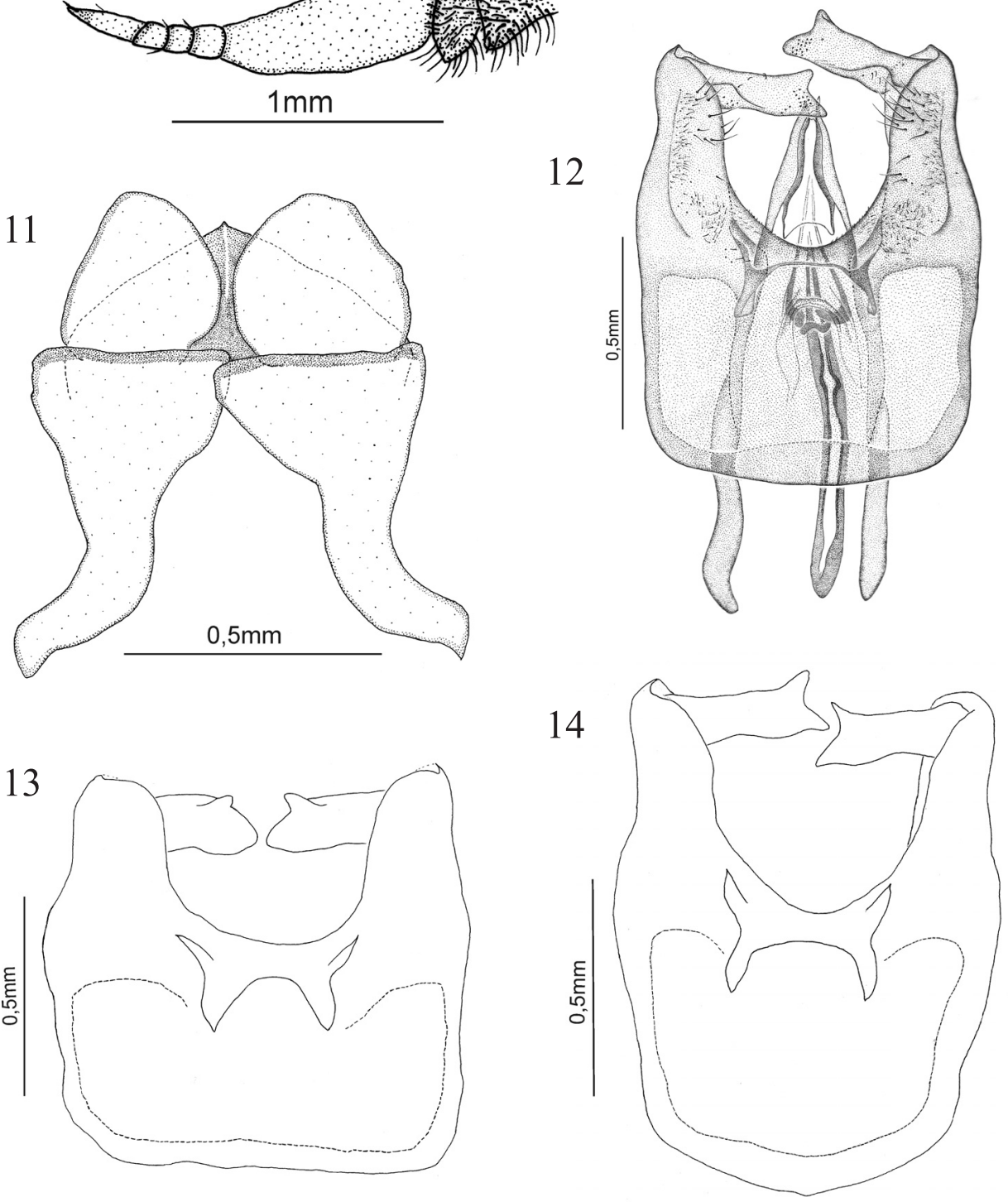

Figuras 10-14. Chlorotabanus flagellatus sp. nov., parátipo macho: 10, antena esquerda, vista lateral; 11, epândrio, cercos e hipoprocto, vista dorsal (cerdas não representadas); 12, hipândrio + gonocoxitos, gonóstilo e edeago, vista ventral; 13, 14, variação do hipândrio + gonocoxitos e gonóstilo, vista ventral (desenhos esquemáticos, cerdas não representadas). 
representado em diversas coleções brasileiras, entretanto, erros de identificação de espécies são constantes, devido à aparente semelhança entre algumas espécies e ao aumento significativo do material coletado desde a última revisão, feita por PHILIP \& FAIRChILD (1956). Dessa forma, Chlorotabanus flagellatus sp. nov. já havia sido identificada algumas vezes como Chlorotabanus inanis (Fabricius, 1787), esta de captura mais frequente e amplamente distribuída no Neotrópico.

Chlorotabanus inanis pode ser diferenciada de $C$. flagellatus sp. nov., pois apresenta placa basal mais curta e com o ângulo dorsal mais elevado, I.F. não atinge 8.0, além das diferenças de pruinosidade e pilosidade. Chlorotabanus flagellatus sp. nov. difere prontamente de C. fairchildi Wilkerson, 1979 e C. mexicanus (Linnaeus, 1758), pois ambas possuem fortes manchas nos cruzamentos das veias transversais e nos ápices das veias longitudinais. Chlorotabanus crepuscularis (Bequaert, 1926) também possui asa com manchas e ocorre apenas no sul dos Estados Unidos. Chlorotabanus ochreus Philip \& Fairchild, 1956 foi descrito de um espécime único, coletado na serra da Bocaina, Estado de São Paulo. Esta espécie não possui outros registros em trabalhos posteriores, mas pode ser diferenciada de $C$. flagellatus sp. nov. por apresentar fronte paralela, baixa e larga (I.F. 4.0), bem como pelo formato da antena, onde a placa basal e o anuli possuem tamanhos similares. Chlorotabanus parviceps (Kröber, 1934) pode ser distinguida pelo valor do I.F. que não ultrapassa 5.5, pelas tíbias anteriores com pêlos pretos no ápice dorsal, e pelas proporções de placa basal e anuli. Chlorotabanus leucochlorus Fairchild, 1961 possui pruinosidade branco-neve e pilosidade branco-prateado, além de pêlos negros esparsos na notopleura, e I.F. < 7.6.

COSCARÓN (1976) fez a ilustração dos caracteres internos de fêmea e macho de C. parviceps, por comparação é possível observar algumas pequenas diferenças entre as duas espécies: em $C$. parviceps a extremidade distal dos cercos da fêmea é bem mais arredondada, encobrindo praticamente todo o hipoprocto; os lobos laterais da forquilha genital diferem claramente entre as duas espécies, em C. flagellatus sp. nov. a margem externa anterior é arredondada e alongada, enquanto em $C$. parviceps é arredondada mas não apresenta o alongamento; as espermatecas são parecidas, embora em C. flagellatus sp. nov. apresente um afilamento mais acentuado na extremidade distal da cápsula espermática; a forma do hipândrio + gonocoxitos difere pelo estreitamento da porção anterior do hipândrio, e pela distância entre a cavidade basal (vista dorsal) do hipândrio + gonocoxitos e a escavação mediana posterior, a qual parece ser maior em C. parviceps. KRoLow \& HENRIQUES (2008) descreveram a terminália do macho de C. leucochlorus, onde o epândrio e o hipoprocto são muito similares ao de $C$. flagellatus sp. nov. Os cercos diferem moderadamente, pois em C. leucochlorus possuem uma reentrância nas margens laterais posteriores. Hipândrio + gonocoxitos, gonóstilo e edeago são muito semelhantes.

Os trabalhos $o p$. cit. são os únicos que tratam dos caracteres internos em Chlorotabanus, não obstante, foi possível diferenciar algumas estruturas internas entre as espécies, mas a sobreposição de caracteres internos não pode ser descartada, pois estas variações de caracteres possivelmente poderão ser observadas ao longo do gradiente morfológico (caracteres externos e internos) encontrado nas diferentes espécies do gênero.

Dados ecológicos. Os horários de coleta contidos nas etiquetas revelam que $C$. flagellatus sp. nov. procurou repasto sanguíneo em Tapirus terrestris (Linnaeus, 1758) entre os períodos de 5:00 e 7:00h e 17:00 e 19:00h, horários característicos do gênero Chlorotabanus. Os ataques tiveram distribuição pelo corpo do Tapirus, exceto pescoço e cabeça, com maior incidência na porção posterior dorsal (anca).

Agradecimentos. Ao Conselho Nacional de Desenvolvimento Científico e Tecnológico (CNPq), pela concessão da bolsa de mestrado ao primeiro autor.

\section{REFERÊNCIAS BIBLIOGRÁFICAS}

Burger, J. F. 1995. Catalog of Tabanidae (Diptera) of North America North of Mexico. Contributions on Entomology, International 1(1):1-100.

Coscarón, S. 1976. Notas sobre tabánidos argentinos XIV. Sobre los géneros Diachlorus Osten Saken, Stibasoma Schiner, Stypommisa Enderlein, Cryptotylus Lutz y Chlorotabanus Lutz (Diptera). Revista de la Sociedad Entomológica Argentina 35:39-50

Cumming, J. M. 1992. Lactic Acid as an Agent for Macerating Diptera Specimens. Fly Times 8:7.

Fairchild, G. B. 1961. Insecta Amapaensia - Diptera: Tabanidae. Studia Entomologica 4(1-4):433-448.

. 1969. Notes on Neotropical Tabanidae. - XII. Classification and distribution, with keys to genera and subgenera. Arquivos de Zoologia do Estado de São Paulo 17(4):199-255.

1985. Notes on Neotropical Tabanidae (Diptera). XVIII. The Genus Leucotabanus Lutz. Myia 3:299-331.

1986. The Tabanidae of Panama. Contributions of the American Entomological Institute 22(3):1-139.

Fairchild, G. B. \& Burger, J. F. 1994. A catalog of the Tabanidae (Diptera) of the Americas south of the United States. Memoirs of the American Entomological Institute 55:1-249.

Henriques, A. L. 1995. A Coleção de Tabanidae (Insecta: Diptera) do Instituto Nacional de Pesquisas da Amazônia (INPA), Manaus, Amazonas, Brasil. Boletim do Museu Paraense Emílio Goeldi, Série Zoologia, 11(1):57-99.

Henriques, A. L. \& Gorayeb, I. S. 1993. A Coleção de Tabanidae (Insecta: Diptera) do Museu Paraense Emílio Goeldi (MPEG), Belém, Pará, Brasil. Goeldiana Zoologia 20:1-23.

Krolow, T. K. \& Henriques, A. L. 2008. Descrição do macho de Chlorotabanus leucochlorus Fairchild (Diptera, Tabanidae). Revista Brasileira de Entomologia 52(2):269-271.

Lutz, A. 1913. Sobre a systematica dos tabanideos, subfamilia Tabaninae. Brazil Medico 27(45):486-490.

McAlpine, J. F. 1981. Morphology and terminology - Adults. In: McAlpine, J. F.; Peterson, B. V.; Shewell, G. E.; Teskey, H. J.; Vockeroth, J. R. \& Wood, D. M. coords. Manual of Nearctic Diptera. Ottawa, Ontario, Agriculture Canada Monograph, Biosystematics Research Institute. v.1, p.9-63.

Philip, C. B. \& Fairchild, G. B. 1956. American biting flies of the genera Chlorotabanus Lutz and Cryptotylus Lutz (Diptera, Tabanidae). Annals Entomological Society of America 49(4):313-324.

Rafael, J. A. \& Charlwood, J. D. 1981. Atividade crepuscular de Chlorotabanus inanis (Fab.) and Cryptotylus unicolor (Wied.) (Diptera: Tabanidae). Acta Amazonica 11(2):411-413.

Sinclair, B. J.; Cumming, J. M. \& Wood, D. M. 1993. Homology and phylogenetic implications of male genitalia in Diptera - lower Brachycera. Entomologica Scandinavica 24(4):407-432.

Wilkerson, R. C. 1979. Horse flies (Dipt. Taban.) of the Colombian Departments of Choco, Valle and Cauca. Cespedesia 8(31$35): 87-433$. 